\title{
DEBATES
}

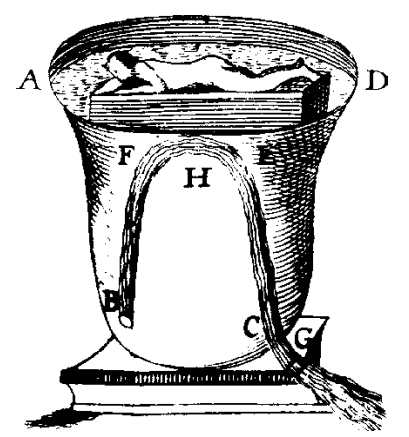

\section{¿TIENE SENTIDO SEGUIR DISTINGUIENDO ENTRE APRENDIZAJE DE CONCEPTOS, RESOLUCIÓN DE PROBLEMAS DE LÁPIZ Y PAPEL Y REALIZACIÓN DE PRÁCTICAS DE LABORATORIO?}

\author{
GIL PÉREZ, DANIEL, ${ }^{1}$ FURIÓ MÁS, CARLES, ${ }^{1}$ VALDÉS, PABLO, ${ }^{2}$ \\ SALINAS, JULIA, ${ }^{3}$ MARTÍNEZ-TORREGROSA, JOAQUÍN, ${ }^{4}$ \\ GUISASOLA, JENARO ${ }^{5}$ GONZÁLEZ, EDUARDO,${ }^{6}$ DUMAS-CARRÉ, ANDRÉE, ${ }^{7}$ \\ GOFFARD, MONIQUE ${ }^{7}$ y PESSOA DE CARVALHO, ANNA M. ${ }^{8}$ \\ ${ }^{1}$ Universitat de València. España. \\ ${ }^{2}$ Instituto Superior Pedagógico EJ Varona. La Habana. Cuba. \\ ${ }^{3}$ Universidad de Tucumán. Argentina. \\ ${ }^{4}$ Universidad de Alicante. España. \\ ${ }^{5}$ Universidad del País Vasco. España. \\ ${ }^{6}$ Universidad de Córdoba. Argentina. \\ ${ }^{7}$ Université París 7. Francia \\ ${ }^{8}$ Universidad de São Paulo. Brasil.
}

\section{RESUMEN}

Nuestra pretensión en este trabajo es cuestionar la distinción clásica entre «teoría», «prácticas de laboratorio» y «problemas». Intentaremos mostrar qué investigaciones realizadas en los tres campos privan de sentido a esta separación que, conviene notar, no guarda paralelismo alguno con la actividad científica real.

\section{SUMMARY}

Our aim with this paper is to question the typical distinction between «theory», «lab» and «paper and pencil problemsolving» activities. We intend to show that research done on these domains does not support their separation in the teaching/learning process. In fact, this separation does not take place in any real scientific activity. 


\section{INTRODUCCIÓN}

La distinción entre «teoría», «prácticas de laboratorio» y «problemas» es aceptada como algo natural en la enseñanza de las ciencias, hasta el punto de que, en los cursos universitarios, dichas actividades son impartidas, muy a menudo, por distintos profesores. Más aún: la propia investigación e innovación en didáctica de las ciencias ha estudiado dichas actividades como líneas de trabajo prácticamente autónomas, tal como pone en evidencia, por ejemplo, el reciente Handbook of Research on Science Teaching and Learning (Gabel, 1994).

Quienes firmamos este documento hemos realizado también investigaciones (y en bastantes casos la tesis doctoral) centradas, por ejemplo, en las prácticas de laboratorio (Gené, 1986, Payá, 1991; González, 1994; Salinas, 1994), la resolución de problemas (Martínez-Torregrosa, 1987; Dumas-Carré, 1987; Ramírez, 1990; Goffard, 1991; Reyes, 1991) o el aprendizaje de conceptos (Solbes, 1986; Carrascosa, 1987; Guisasola, 1996). En ello ha influido, sin duda, el carácter necesariamente acotado de las investigaciones, pero también, al menos inicialmente, el peso de una tradición docente asumida acríticamente. Los resultados de nuestras investigaciones y, en general, todo el desarrollo de la didáctica de las ciencias, nos ha llevado al convencimiento -que intentaremos fundamentar y que sometemos aquí a debate- de que dicha separación no está justificada y puede constituir un serio obstáculo para una efectiva renovación de la enseñanza de las ciencias.

\section{NECESIDAD DE UN REPLANTEAMIENTO GLOBAL}

Durante las últimas décadas hemos asistido a un importante desarrollo de la innovación e investigación en torno a la enseñanza-aprendizaje de las ciencias (Gil, 1996). Prueba de ello es el notable crecimiento del número de trabajos publicados (Pfundt y Duit, 1993) así como el de revistas específicas existentes, tesis doctorales realizadas, etc. (Cudmani y Pesa, 1993; Gil, 1994).

No obstante, como ya hemos señalado, las investigaciones sobre los distintos aspectos del proceso de enseñanza-aprendizaje se han llevado a cabo, hasta recientemente, de forma autónoma y con ritmos muy diferentes. Así, en la década de los ochenta, la investigación se centró en el estudio de las concepciones alternativas de los estudiantes (Pfundt y Duit, 1994), mientras otros aspectos fueron mucho menos estudiados y algunos, como la evaluación, prácticamente ignorados. Pero lo que queremos subrayar, sobre todo, es que los estudios sobre preconcepciones tenían poco que ver, en general, con los realizados, por ejemplo, sobre las prácticas de laboratorio, o éstos con los relativos a la resolución de problemas de lápiz y papel o la evaluación. Es preciso reconocer, pues, que la didáctica de las ciencias constituía un campo de estudios relativamente dispersos o bien, como afirmaban Klopfer (1983) o Tiberghien (1983), un dominio preteórico.
Por otra parte, la transferencia de estos desarrollos a la práctica docente ha sido más limitada de lo que era de desear. Como ha señalado Briscoe (1991), cada año miles de profesores asisten a cursos o participan en encuentros con la intención de perfeccionarse profesionalmente y poder utilizar nuevas técnicas, nuevos materiales curriculares, nuevas formas de favorecer el aprendizaje de sus alumnos y alumnas. Sin embargo, muchos de estos profesores y profesoras se encuentran, antes de que puedan darse cuenta, enseñando de la misma forma en que lo habían hecho siempre, adaptando los nuevos materiales o técnicas a los patrones tradicionales. Se genera así una lógica frustración y decepción al percibir que las cosas no han funcionado mejor que los años precedentes, a pesar de las nuevas y prometedoras ideas. En nuestra opinión, este resultado no es debido, en general, a que las innovaciones contempladas en los cursos de formación carezcan de interés, sino que pone en evidencia que un modelo de enseñanza (incluso la denostada «enseñanza tradicional») es algo más que un conjunto de elementos dispersos e intercambiables: posee una cierta coherencia y cada uno de sus elementos viene apoyado por los restantes (Viennot, 1989; Gil, 1991). Dicho con otras palabras: la transformación efectiva de la enseñanza habitual de las ciencias precisa algo más que el simple reconocimiento de algunas de sus carencias más visibles o que la introducción de innovaciones puntuales, restringidas a un sólo aspecto.

Se ha ido así imponiendo la conciencia de que los tratamientos puntuales, inconexos, resultan ineficaces y de que se precisa un replanteamiento global de todo el proceso de enseñanza-aprendizaje de las ciencias que integre coherentemente distintos aspectos hasta aquí estudiados separadamente. Ello ha conducido, estos últimos años, a un significativo cambio en el desarrollo de la investigación e innovación didáctica, hasta el punto de que, como afirma Hodson (1992): «Hoy es ya posible construir un cuerpo de conocimientos en el que se integren coherentemente los distintos aspectos relativos a la enseñanza-aprendizaje de las ciencias.»

Naturalmente, este cambio desde una situación preteórica a la conformación de un cuerpo coherente de conocimientos constituye un proceso complejo, que no podemos analizar aquí detalladamente, pero en el que queremos destacar los siguientes aspectos:

- El consenso creciente en tomo a las propuestas constructivistas, es decir, en torno a la idea de que un aprendizaje significativo de los conocimientos científicos requiere la participación de los estudiantes en la (re)construcción de los conocimientos que habitualmente se transmiten ya elaborados. La reciente investigación didáctica, tanto en el campo de las preconcepciones como en el de los trabajos prácticos, la resolución de problemas, etc. está mostrando que «los estudiantes desarrollan mejor su comprensión conceptual y aprenden más acerca de la naturaleza de la ciencia cuando 


\section{DEBATES}

participan en investigaciones científicas, con tal que haya suficientes oportunidades y apoyo para la reflexión» (Hodson, 1992).

Ello no supone -como hemos intentado dejar claro en diversos trabajos (Gil et al., 1993b)- volver a la desacreditada propuesta del «aprendizaje por descubrimiento»: es preciso subrayar que ahora no se concibe a los alumnos y alumnas como investigadores autónomos trabajando en la frontera del conocimiento. Esta metáfora (que fue propuesta como reacción a la que concebía a los estudiantes como simples receptores) presenta graves limitaciones y no resulta útil para organizar el trabajo de los alumnos. Una metáfora que conciba a los estudiantes como investigadores noveles (que, estructurados en equipos cooperativos, abordan situaciones problemáticas de interés, interaccionando con los otros equipos y con el resto de la comunidad científica, representada por el profesor y los textos) permite, en nuestra opinión, una mejor comprensión de la situación de aprendizaje escolar.

- La efectividad de una orientación del aprendizaje como investigación dirigida (que acabamos de resumir) exige la superación de los reduccionismos y visiones deformadas de la naturaleza de la ciencia que impregnan la epistemología espontánea del profesorado y que fueron la causa, en gran medida, del fracaso de la orientación de «aprendizaje por descubrimiento». Como Bell y Pearson (1992) han puesto de relieve, empieza a comprenderse que, si se quiere cambiar lo que los profesores y los estudiantes hacemos en las clases de ciencias, es preciso previamente modificar la epistemología de los profesores y salir al paso, en particular, de visiones deformadas sobre el trabajo científico que actúan como auténticos obstáculos.

La investigación sobre el pensamiento docente espontáneo se ha convertido, efectivamente, a principios de esta década, en uno de los factores que más están contribuyendo a la renovación de la enseñanza de las ciencias, al hacer posible el cuestionamiento de numerosas concepciones (ideas, comportamientos y actitudes) en tomo a la naturaleza de la ciencia y a su enseñanza y aprendizaje, que los profesores y profesoras hemos adquirido por «impregnación ambiental» y que tomamos como expresión de «lo natural», escapando así a la crítica (Gil et al., 1991; Porlan, 1993; Désautels et al., 1993; Hodson, 1993; Meichstry, 1993; Gil y Pessoa, 1994; Praia y Cachapuz, 1994; Duschl, 1995; Porlan y Martín, 1996).

Conviene notar a este respecto que, aunque las críticas a las concepciones del profesorado sobre la ciencia han sido objeto de estudio desde hace décadas, estas críticas se limitaban, en general, a denunciar las concepciones inductivistas y las visiones rígidas, algorítmicas, del llamado «método científico». Ahora, sin embargo, la crítica se extiende a otras deformaciones igualmente comunes (Gil, 1993b y 1997; Fernández, 1995; Orozco, 1995) que, por acción u omisión transmite la enseñanza (visión aproblemática, exclusivamente analítica, individualista, socialmente neutra...). Debemos añadir, sin embargo, que, si bien dichas preconcepciones docentes son muy abundantes y constituyen serios obstáculos (en la medida en que son aceptadas acríticamente), basta favorecer una reflexión colectiva con un mínimo de profundidad para que los profesores y profesoras - gracias al distanciamiento crítico que supone adoptar una actitud investigadora- cuestionemos estas deformaciones y reduccionismos, realizando análisis cuyos resultados son coincidentes, en buena medida, con los obtenidos por la investigación didáctica y con los análisis contemporáneos de la epistemología científica.

Se han ido abriendo así serias perspectivas para un replanteamiento global de la enseñanza de las ciencias, superando los obstáculos que representaban las concepciones docentes espontáneas y, muy en particular, la idea de que la enseñanza es algo trivial que puede abordarse «con algo de experiencia y sentido común». Dicho con otras palabras: comienza a ser posible avanzar en la solución de los problemas que plantea la enseñanza de las ciencias en la medida misma en que dicha enseñanza es contemplada como situación problemática que exige investigación, es decir, cuestionamiento de las «evidencias», de «lo que siempre se ha hecho». Reencontramos así un hecho bien conocido en la historia del desarrollo científico: las mayores dificultades con que, a menudo, ha tropezado el desarrollo de una ciencia, han derivado de supuestos implícitos, aceptados sin cuestionamiento alguno como algo «evidente», escapando así a la crítica. En tales casos se impone -como la historia de las ciencias ha mostrado reiteradamente- un replanteamiento en profundidad que analice hasta (o, mejor, sobre todo) aquello que parece más seguro. Es esta actitud de análisis crítico de las «evidencias» y de estudio detenido de los problemas, la que ha permitido, insistimos, avanzar en la búsqueda de soluciones a dichos problemas y, en definitiva, en el replanteamiento global de la enseñanza de las ciencias. La orientación de los distintos aspectos del proceso de enseñanza-aprendizaje de las ciencias (desde la introducción y manejo de conceptos a la evaluación, pasando por las prácticas de laboratorio y la resolución de problemas) ha ido experimentando una profunda transformación que apunta hacia la consolidación de un nuevo modelo. Sin embargo, estos distintos aspectos han seguido siendo contemplados, en general, como elementos autónomos, y tanto las investigaciones como las propuestas de innovación derivadas continúan abordando aisladamente cada uno de estos aspectos (Gabel, 1994).

Nuestra pretensión en este trabajo es, precisamente, cuestionar la distinción clásica entre teoría, prácticas y problemas. Intentaremos mostrar que la orientación del aprendizaje como una investigación dirigida priva de sentido a esta separación que, conviene notar, no guarda paralelismo alguno con la actividad científica real. En la medida en que pretendamos proporcionar a los estudiantes una visión correcta del trabajo científico, el tratamiento por separado de aspectos (la teoría, las prácticas y los problemas) que en la actividad científica aparecen absolutamente imbricados, se convierte en un factor distorsionante, es decir, en un obstáculo. Hemos de reconocer, sin embargo, que esta elemental consideración no ha bastado para rechazar claramente esta 
compartimentación de la actividad escolar. Ello constituye, pensamos, un ejemplo más del peso de tradiciones asumidas acríticamente. Afortunadamente, los avances realizados por la investigación e innovación didácticas, en cada uno de los tres campos, ha ido mostrando convergencias que reclaman su integración en un único proceso. A continuación nos referiremos brevemente a estas convergencias.

\section{LA TRANSFORMACIÓN DE LAS PRÁCTI- CAS DE LABORATORIO}

La idea de buscar en la realización de abundantes trabajos prácticos la superación de una enseñanza puramente libresca y la solución a las dificultades en el aprendizaje de las ciencias cuenta con una larga tradición (Lazarowitz y Tamir, 1994). De hecho constituye una intuición básica de la generalidad de los profesores de ciencias, que contemplan el paso a una enseñanza eminentemente experimental como una especie de «revolución pendiente» (Gil et al., 1991), permanentemente dificultada, en la mayoría de los países, por factores externos (falta de instalaciones y material adecuado, excesivo número de alumnos, carácter enciclopédico de los currículos...). La influencia de esta tendencia ha sido particularmente notable en el mundo anglosajón, donde en los años sesenta y setenta se elaboraron y pusieron en práctica numerosos proyectos de aprendizaje «por descubrimiento autónomo», centrados en el trabajo experimental y en «los procesos de la ciencia» (olvidándose los contenidos). No podemos detenernos aquí en el análisis de las serias limitaciones de estas propuestas de aprendizaje (Ausubel, 1978; Gil, 1983; Millar y Driver, 1987; Sanmartí et al., 1990; Salinas y Cudmani, 1992...). Y, aunque la orientación más general de los trabajos prácticos es la que los concibe como mera ilustración de los conocimientos teóricos introducidos, numerosos autores han destacado que, en ambos casos, las prácticas de laboratorio aparecen como «recetas» que transmiten una visión deformada y empobrecida de la actividad científica (Hodson, 1985 y 1992b; Gené, 1986; Gil y Payá, 1988; Gil et al., 1991; Payá, 1991; González, 1994; Salinas, $1994 \ldots)$.

Debemos señalar, sin embargo, que, cuando se favorece una discusión detenida de la naturaleza del trabajo científico, los mismos profesores que conciben las prácticas de laboratorio como simples recetas ilustrativas, ven la necesidad de concebirlas orgánicamente vinculadas al tratamiento de un problema relevante, a la construcción de hipótesis que focalicen la investigación, a la invención de diseños experimentales, etc., incorporando aspectos clave de la actividad científica habitualmente ignorados (Gil et al., 1991).

Podría concluirse que existe una disposición positiva para considerar las prácticas de laboratorio como ocasión de familiarizar a los estudiantes con el trabajo científico y que resulta relativamente simple conseguir que los profesores cuestionen las prácticas «receta»y hagan suyas propuestas que ofrecen una visión más correcta de la ciencia (Gené, 1986; Payá, 1991; González, 1994; Salinas, 1994). Sin embargo, esa relativa facilidad para trasformar los trabajos prácticos sigue escondiendo, en nuestra opinión, una visión reduccionista de la actividad científica, que asocia prioritariamente investigación a trabajo experimental, y que ha actuado como obstáculo en la renovación de otros aspectos del proceso de enseñanza-aprendizaje de las ciencias.

Nos referiremos a ello al ocuparnos, a continuación, de las aportaciones de la investigación didáctica en torno a la resolución de problemas de lápiz y papel.

\section{LA TRANSFORMACIÓN DE LOS PROBLE- MAS DE LAPIZ Y PAPEL}

La asociación de las prácticas de laboratorio con el trabajo científico responde, como ya hemos indicado, a una intuición básica, tanto de los profesores como de los propios estudiantes, y ello facilita, sin duda, la superación de las prácticas «receta»y su enriquecimiento, con la inclusión de aspectos clave de la actividad científica como la construcción de hipótesis, etc. La transformación de los problemas de lápiz y papel, sin embargo, ha tropezado con dificultades muy superiores, pese a la abundante investigación realizada en este campo (recordemos, por ejemplo, que en el Handbook of Research on Science Teaching and Learning (Gabel, 1994), de un total de 19 capítulos, 6 (!) están dedicados a la resolución de problemas).

Hemos analizado el origen de estas dificultades en otros trabajos (Gil y Martínez-Torregrosa, 1983; Gil, Martínez-Torregrosa y Senent, 1988; Gil, Dumas-Carré et al., 1990; Gil y Pessoa, 1994) y son muy numerosas las tesis doctorales que recientemente se han centrado en «el problema de los problemas» (Martínez-Torregrosa, 1987; Dumas-Carré, 1987; Ramírez, 1990; Goffard, 1990; Reyes, 1991; Oñorbe, 1993...). Nos limitaremos aquí a recordar que uno de los principales obstáculos estriba en el hecho de que, en realidad, no se enseña $a$ resolver problemas, es decir, a enfrentarse a situaciones desconocidas, ante las cuales el resolvente se siente inicialmente perdido, sino que los profesores explicamos soluciones que nos son perfectamente conocidas y que, por supuesto, no nos generan ningún tipo de dudas ni exigen tentativas. La pretensión del profesor es que el estudiante vea con claridad el camino a seguir; dicho con otras palabras, pretendemos convertir el problema en un no-problema. Consecuentemente, los estudiantes pueden aprender dicha solución y repetirla ante situaciones prácticamente idénticas, pero no aprenden a abordar un verdadero problema y cualquier pequeño cambio les supone dificultades insuperables provocando manipulaciones no significativas de datos, fórmulas e incógnitas y, muy a menudo, el abandono.

Sin embargo, la cuestión «¿Qué hemos de entender por problema?» permite iniciar un proceso de clarificación que desbloquea la renovación de la didáctica de la 
resolución de problemas (Gil, Martínez-Torregrosa y Senent, 1988; Garrett et al., 1990; Gil y Pessoa, 1994). En efecto, si se acepta la idea de que todo problema es una situación ante la cual se está inicialmente perdido, resulta lógico preguntarse, para enfocar la resolución de problemas científicos, qué hacen los científicos cuando se enfrentan con lo que para ellos constituye un verdadero problema y no ante un enunciado de lápiz y papel como los que se incluyen en los libros de texto. Se puede esperar, en efecto, que, delante de problemas de lápiz y papel, los científicos -que son a menudo profesoresadopten actitudes características de la enseñanza habitual y consideren los problemas como situaciones que se debe saber resolver y no como verdaderos problemas. En este sentido, los estudios hechos sobre la manera en que los «expertos» abordan los «problemas» de lápiz y papel estarían todavía muy lejos de lo que supone enfrentarse a un verdadero problema. Es, pues, más útil preguntarse qué es lo que los científicos hacen cuando tienen que habérselas con auténticos problemas para ellos. La respuesta en este caso es «simplemente» que se comportan como investigadores, implicándose en una actividad compleja y creativa de razonamiento en términos de hipótesis (Gil et al., 1991). Pero antes de intentar precisar lo que supondría un enfoque investigativo en la resolución de problemas de lápiz y papel, cabe preguntarse cuál es la razón de que habitualmente no se piense en un enfoque como ése cuando se plantean dichos problemas. Con otras palabras, ¿qué es lo que en los enunciados habituales puede dificultar un tratamiento científico de los problemas, hasta el punto de no hacer pensar ni siquiera en la conveniencia de construir alguna hipótesis orientadora?

Una pregunta como ésta lleva a comprender que la inclusión de los datos en el enunciado, como punto de partida, orienta la resolución hacia el manejo de unas determinadas magnitudes sin que ello responda a una reflexión cualitativa ni a las subsiguientes hipótesis. De este modo, al resolver un problema, el alumno se ve abocado a buscar aquellas ecuaciones que pongan en relación los datos e incógnitas proporcionados en el enunciado, cayendo así en un puro operativismo. No basta, pues, denunciar dicho operativismo: se trata de hacerlo imposible atacando sus causas. La comprensión de que la presencia de los datos en el enunciado, así como la indicación de todas las condiciones existentes -todo ello como punto de partida- responden a concepciones inductivistas y orientan incorrectamente la resolución (los científicos han de buscar los datos que consideran pertinentes, ¡no los encuentran encima de la mesa!) constituye un paso esencial en el desbloqueo de la enseñanza habitual de problemas y sus limitaciones. Pero al mismo tiempo genera desconcierto, porque choca con la práctica reiterada, con lo que «siempre» se ha hecho. Un enunciado sin datos, se señala, ¿no será algo excesivamente ambiguo frente a lo cual los alumnos acaben extraviándose? Ahora bien, la ambigüedad o, dicho con otras palabras, las situaciones abiertas, ¿no son acaso una característica esencial de las situaciones genuinamente problemáticas? ¿Y no es también una de las tareas fundamentales del trabajo científico acotar los problemas abiertos, imponer condiciones simplificatorias?
Otra dificultad que suele apuntarse durante esta discusión se refiere a la posibilidad de eliminar los datos y precisiones de los enunciados habituales y construir enunciados más abiertos capaces de generar una resolución acorde con las características del trabajo científico. A este respecto, el trabajo realizado en numerosos talleres y cursos de perfeccionamiento del profesorado ha permitido constatar que los enunciados habituales son «traducibles» sin dificultad. Así, por ejemplo, el enunciado:

Sobre un móvil de $5.000 \mathrm{~kg}$, que se desplaza con una velocidad de $20 \mathrm{~m} / \mathrm{s}$, actúa una fuerza de frenado de 10.000 N. ¿Qué velocidad llevará a los $75 \mathrm{~m}$ de donde comenzó a frenar?

puede ser traducido a una situación más abierta y que no señale cuáles son las magnitudes relevantes, como la siguiente:

Un coche comienza a frenar cuando el chófer ve la luz amarilla. ¿Qué velocidad llevará al llegar al semáforo?

Por supuesto, son posibles distintos enunciados, distintas situaciones problemáticas, más o menos abiertas; así, el problema anterior puede dar lugar, entre otros muchos, a este enunciado que, aunque aparentemente diferente, plantea una situación muy similar:

\section{¿Chocará el tren contra la roca caída en la vía?}

De hecho, cuando se plantea a varios grupos la traducción de un mismo enunciado tradicional, se obtienen distintas propuestas de situaciones problemáticas, en general igualmente válidas. En cualquier caso interesa destacar que estas traducciones no plantean dificultades mayores y que cualquier enunciado habitual es transformable en situación problemática (Gil y MartínezTorregrosa, 1987). Por otra parte subsiste la cuestión de cómo orientar a los alumnos para abordar dichas situaciones, puesto que no basta, obviamente, con enfrentarles a enunciados sin datos para lograr una actividad exitosa.

No podemos detenemos aquí a describir y fundamentar la propuesta de resolución de problemas como investigación que se deriva de estos planteamientos y que ha sido calificada, en el Handbook of Research on Science Teaching and Learning, como "an interesting alternative approach to problem solving» (Maloney, 1994). Señalaremos tan sólo que la cuestión de qué orientaciones habrá que proporcionar a los estudiantes para abordar la resolución de problemas sin datos (con lo que ya no es posible el simple juego de datos, fórmulas e incógnitas) conduce, tras una mínima clarificación de qué entender por trabajo científico, a avanzar propuestas similares a las que resumimos en el cuadro I.

Conviene hacer hincapié en que las orientaciones resumidas en dicho cuadro no constituyen un algoritmo que pretenda guiar paso a paso la actividad de los estudiantes. Muy al contrario, se trata de indicaciones genéricas destinadas a llamar la atención contra ciertos «vicios 


\section{DEBATES}

Cuadro I

Aspectos esenciales en la resolución de problemas como investigación.

- Discutir cuál puede ser el interés de la situación problemática abordada. Esta discusión, además de favorecer una actitud más positiva, permitiendo una aproximación funcional a las relaciones CTS, contribuye a proporcionar una concepción preliminar de la tarea, evitando que los estudiantes se vean sumergidos en el tratamiento de una situación sin haber podido siquiera formarse una primera idea motivadora.

- Realizar un estudio cualitativo de la situación, intentando acotar y definir de manera precisa el problema, tomando decisiones sobre las condiciones que se consideran reinantes, etc.

- Emitir hipótesis fundadas sobre los factores de los que puede depender la magnitud buscada y sobre la forma de esta dependencia, imaginando, en particular, casos límite de fácil interpretación física.

- Elaborar y explicitar posibles estrategias de resolución (en plural) antes de proceder a ésta, para posibilitar una contrastación rigurosa de las hipótesis y mostrar la coherencia del cuerpo de conocimientos de que se dispone.

- Realizar la resolución verbalizando al máximo, fundamentando lo que se hace y evitando, una vez más, el puro ensayo y error u operativismos carentes de significación física. Conviene, en general, comenzar con una resolución literal (que permite mantener el tratamiento próximo a los principios manejados y facilita el análisis de los resultados). Dicha resolución literal puede completarse después solicitando los datos correspondientes al profesor (o, mejor, introduciendo valores plausibles de los mismos).

- Analizar cuidadosamente los resultados a la luz del cuerpo de conocimientos y de las hipótesis elaboradas y, en particular, de los casos límite considerados.

- Considerar las perspectivas abiertas por la investigación realizada, contemplando, por ejemplo, el interés de abordar la situación a un nivel de mayor complejidad, sus implicaciones teóricas (profundización en la comprensión de algún concepto) o prácticas (aplicaciones técnicas) Concebir, muy en particular, nuevas situaciones a investigar, sugeridas por el estudio realizado.

- Elaborar una memoria que explique el proceso de resolución y que destaque los aspectos de mayor interés en el tratamiento de la situación considerada. Incluir, en particular, una reflexión global sobre lo que el trabajo puede haber aportado, desde el punto de vista metodológico u otro, para incrementar la competencia de los resolventes.

metodológicos» connaturales: la tendencia a caer en operativismos ciegos o a pensar en términos de certeza, lo que se traduce en no buscar posibles caminos alternativos de resolución, en no poner en duda y analizar cuidadosamente los resultados, etc.

Una reorientación de la resolución de problemas como la que aquí se propone ha sido ya utilizada sistemáticamente por numerosos profesores con resultados muy positivos, tanto en lo que respecta a la mejora de la capacidad de los estudiantes para enfrentarse a las situaciones problemáticas (incluidos los problemas estándar de la enseñanza habitual), como en lo que se refiere a su interés por la resolución de problemas, que les resulta ahora una actividad mucho más creativa y satisfactoria (Gil, Martínez-Torregrosa y Senent, 1988; Furió, Iturbe y Reyes, 1994).
Pero lo que aquí queremos resaltar, sobre todo, es el hecho de que una propuesta como la que acabamos de resumir se asemeja notablemente a la orientación dada a las prácticas de laboratorio como investigación dirigida. Puede pensarse, pues, en abrazar las prácticas de laboratorio y la resolución de problemas de lápiz y papel como variantes de una misma actividad: el tratamiento de situaciones problemáticas abiertas, con una orientación próxima a lo que constituye el trabajo científico. De hecho, la puesta a prueba de una hipótesis, en una investigación real, puede y debe hacerse tanto experimentalmente como mostrando la coherencia de sus implicaciones con el cuerpo de conocimientos aceptado por la comunidad científica.

Se diluyen así las marcadas diferencias que, en la enseñanza por transmisión de conocimientos ya elaborados, 
se dan entre las prácticas de laboratorio y los problemas de lápiz y papel. Pero es posible ir más allá en este proceso de integración, extendiéndolo a lo que en la jerga docente denominamos la teoría. Intentaremos mostrar esto en el siguiente apartado.

\section{LA TRANSFORMACIÓN DEL APRENDIZA- JE CONCEPTUAL}

No vamos a insistir aquí en la importancia de la orientación constructivista para el aprendizaje de conceptos. Tampoco creemos necesario extendemos en la descripción de las características esenciales de la nueva visión del aprendizaje de las ciencias, expuestas con detalle por diversos autores (Posner et al., 1982; Osborne y Wittrock, 1983; Driver, 1986...). Tan sólo llamaremos la atención sobre los esfuerzos realizados recientemente para profundizar en dichas propuestas y salir al paso de interpretaciones simplistas de las mismas.

Como Carretero y Limón (1996) indican, «en algunos ámbitos educativos se ha ofrecido una imagen demasiado fácil y estereotipada del constructivismo. Dicha imagen suele consistir en la convicción, más bien estólida, de que la aplicación de fórmulas del tipo tomemos los conocimientos previos de los alumnos, planteémosles conflictos cognitivos y modifiquémoslos solucionará fácilmente numerosos problemas educativos». Es cierto que dicha estrategia puede, puntualmente, dar resultados muy positivos al llamar la atención sobre el peso de ciertas ideas de sentido común, asumidas acríticamente como evidencias. Pero también es cierto, que, practicada, de forma reiterada, produce una inhibición y un rechazo muy comprensibles. En efecto, ¿qué sentido tiene hacer que los alumnos expliciten y afiancen sus ideas para seguidamente cuestionarlas? ¿Cómo no ver en ello un artificio que aleja la situación de lo que constituye el objetivo central de la construcción de conocimientos? Esa construcción nunca se plantea para cuestionar ideas, para provocar cambios conceptuales, sino para resolver problemas de interés para los investigadores (es decir, en nuestro caso para los alumnos); problemas que se abordan, como es lógico, a partir de los conocimientos que se poseen y de nuevas ideas que se construyen a título tentativo. En ese proceso, las concepciones iniciales podrán experimentar cambios e incluso, aunque más raramente, ser cuestionadas radicalmente, pero ése no será nunca el objetivo, sino, repetimos, la resolución de los problemas planteados (Gil et al., 1991).

Ello concede un estatus muy diferente a las situaciones de conflicto cognoscitivo: ya no suponen para los alumnos el cuestionamiento externo de las ideas personales, ni la reiterada aceptación de las insuficiencias del propio pensamiento (con las consiguientes implicaciones afectivas), sino un trabajo de profundización en el que unas ideas (tomadas como hipótesis) son sustituidas por otras (tan propias como las anteriores) (Cudmani, Salinas y Pesa, 1991).
No se trata, como puede verse, de eliminar los conflictos cognoscitivos, sino de evitar que adquieran el carácter de una confrontación entre las ideas propias y los conocimientos científicos. A este respecto Solomon (1991) argumenta que «tras impulsar la expresión de un conjunto de opiniones particulares, el profesor no puede simplemente rechazar las que no se ajustan a la teoría vigente. De ese modo dejaría de ser posible un diálogo abierto».

Por todo ello, la estrategia de enseñanza que nos parece más coherente con la orientación del aprendizaje como construcción de conocimientos científicos es la que asocia el aprendizaje al tratamiento de situaciones problemáticas abiertas (Furió y Gil, 1978; Driver y Oldham, 1986; Gil y Martínez-Torregrosa, 1987; Burbules y Linn, 1991; Gil et al., 1991; Wheatley, 1991; Gil, 1993; Furió, 1994) que puedan generar el interés de los estudiantes. Un esquema de esta estrategia de enseñanza queda reflejada en el cuadro II.

En las cuatro fases que esquematizan la estrategia de enseñanza propuesta en dicho cuadro hemos intentado integrar aspectos esenciales que afectan a la actividad científica y que han sido reiteradamente resaltados por la historia y la filosofía de la ciencia, pero que a menudo no son suficientemente tenidos en cuenta en la enseñanza de las ciencias. Nos referimos concretamente a los problemas de contextualización del trabajo científico (relaciones CTS, toma de decisiones...) y a los componentes afectivos (interés por la tarea, clima de trabajo...) (Solbes y Vilches, 1989; Simpson et al., 1994; Berger et al., 1994; Jiménez, 1996).

El aprendizaje de las ciencias es concebido así no como un simple cambio conceptual, sino como un cambio a la vez conceptual, metodológico y actitudinal. Ello implica, por otra parte, como evidencia el cuadro II (cuyas semejanzas con el cuadro I merecen ser resaltadas), una completa integración de la «teoría», las «prácticas» y los «problemas» en un proceso único de construcción de conocimientos científicos.

\section{CONCLUSIÓN}

La convergencia de las investigaciones realizadas en tomo a las prácticas de laboratorio, los problemas de lápiz y papel y el aprendizaje conceptual se convierte, como hemos intentado mostrar, en un fuerte apoyo a las propuestas de aprendizaje de las ciencias como un proceso de investigación dirigida. Dicha convergencia cuestiona, por otra parte, la separación clásica entre «teoría», «prácticas» y «resolución de problemas». Se rompe así con un tratamiento separado de actividades que en la investigación científica aparecen absolutamente imbricadas y cuya persistencia en la enseñanza contribuye a transmitir una visión deformada de la ciencia. De hecho, los intentos realizados de plantear el aprendizaje de dominios científicos concretos (mecánica, etc.) como una construcción de conocimientos está llevando a una integración funcional de dichas actividades, sin que sea 
1. Plantear situaciones problemáticas que -teniendo en cuenta las ideas, la visión del mundo, las destrezas y las actitudes de los alumnos y alumnas-generen interés y proporcionen una concepción preliminar de la tarea.

2. Proponer a los estudiantes el estudio cualitativo de las situaciones problemáticas planteadas y la toma de decisiones para acotar problemas precisos (ocasión para que comiencen a explicitar funcionalmente sus ideas) y comenzar a concebir un plan para su tratamiento.

3. Orientar el tratamiento científico de los problemas planteados, lo que conlleva, entre otros:

- La emisión de hipótesis, incluida la invención de conceptos, la elaboración de modelos... (ocasión para que las ideas previas sean utilizadas para hacer predicciones).

- La elaboración de estrategias (incluyendo, en su caso, diseños experimentales) para la contrastación de las hipótesis a la luz del cuerpo de conocimientos de que se dispone.

- La realización de las estrategias y el análisis de los resultados, considerando las predicciones de las hipótesis, cotejándolos con los obtenidos por otros grupos de alumnos y por la comunidad científica, estudiando su coherencia con el cuerpo de conocimientos... Ello puede convertirse en ocasión de conflicto cognoscitivo entre distintas concepciones (tomadas todas ellas como hipótesis) y obligar a concebir nuevas hipótesis.

4. Plantear el manejo reiterado de los nuevos conocimientos en una variedad de situaciones para hacer posible la profundización y afianzamiento de los mismos, poniendo un énfasis especial en las relaciones Ciencia/Tecnología/Sociedad que enmarcan el desarrollo científico (propiciando, a este respecto, la toma de decisiones) y dirigiendo todo este tratamiento a mostrar el carácter de cuerpo coherente que tiene toda ciencia.

Favorecer, en particular, las actividades de síntesis (esquemas, memorias, recapitulaciones, mapas conceptuales...), la elaboración de productos (susceptibles de romper con planteamientos excesivamente escolares y de reforzar el interés por la tarea) y la concepción de nuevos problemas.

posible distinguir entre teoría, prácticas o problemas (Calatayud et al., 1990; Azcona et al., 1992; MartínezTorregrosa et al., 1993 y 1995; Gil y Valdés, 1995; Guisasola et al., 1996; National Research Council, 1996; McDermott, 1996...).

Hemos de añadir que esta integración ha comenzado a enriquecerse últimamente con aportaciones de investigaciones e innovaciones relativas al uso de los ordenadores. Éste es un aspecto que merece una atención particular, puesto que supone la incorporación de los cambios metodológicos que ha originado la utilización de los ordenadores en la actividad científica (Valdés y Valdés, 1994), pero que, por razones de espacio, no podemos desarrollarlo aquí, por lo que nos limitaremos a remitirnos a algunos ejemplos que muestran «en acto»

\section{REFERENCIAS BIBLIOGRÁFICAS}

AUSUBEL, D.P. (1978). Psicología educativa. Un punto de vista cognoscitivo. México: Trillas.

BELL, B.F. y PEARSON, J. (1992). Better Learning. International Journal of Science Education, 14(3), pp. 349-361.

BRISCOE, C. (1991). The dynamic interactions among beliefs, role methaphores and teaching practices. A case study of teacher change. Science Education, 75(2), pp. 185-199. las virtualidades de dicha incorporación (Gil y Valdés, 1995 y 1997).

Digamos, por último, que investigaciones centradas en la evaluación de la enseñanza-aprendizaje de las ciencias, a las que aquí nos remitimos simplemente (Cudmani, Pesa y Salinas, 1986; Alonso, Gil y MartínezTorregrosa, 1992a, 1992b, 1995; Jorba y Sanmartí, 1993...), cuestionan la consideración de la evaluación como una actividad «especial», separada del proceso de enseñanza-aprendizaje. Tampoco podemos entrar aquí, por razones de espacio, en la discusión de esta cuestión fundamental, pero sí señalaremos que esta integración de la evaluación apunta también hacia un planteamiento del aprendizaje de las ciencias más coherente con la actividad científica. 
CARRASCOSA, J. (1987). Tratamiento didáctico, en la enseñanza de las ciencias, de los errores conceptuales. Tesis doctoral. València: Servei de Publicacions de la Universitat de València.

CARRETERO, M. y LIMÓN, M. (1996). Problemas actuales del constructivismo. De la teoría a la práctica, en Rodrigo, M.J. Rodrigo y Arnay, J. (eds.). La construcción del conocimiento escolar. Ecos de un debate.

CUDMANI, L. y PESA, M. (1993). La integración de aportes interdisciplinares en la generación de una nueva disciplina. Revista de Enseñanza de la Física, vol. extra.

CUDMANI, L., SALINAS, J. y PESA, M. (1991). La generación autónoma de «conflictos cognoscitivos» para favorecer cambios de paradigmas en el aprendizaje de la física. Enseñanza de las Ciencias, 9 (3), pp. 237-242.

DÉSAUTELS, J., LAROCHELLE, M., GAGNÉ, B. y RUEL. F. (1993). La formation a l'enseignement des sciences: le virage épistémologique. Didaskalia, 1, pp. 49-67.

DRIVER, R. (1986). Psicología cognoscitiva y esquemas conceptuales de los alumnos. Enseñanza de las Ciencias, 4(1), pp. 3-15.

DRIVER, R. y OLDHAM, V. (1986). A constructivist approach to curriculum development in science. Studies in Science Education, 13, pp. 105-122.

DUMAS-CARRÉ, A. (1987). La resolution de problemes en Physique au Lycée. Tesis doctoral. Universidad de París 7.

DUSCHL, R. (1995). Más allá del conocimiento: los desafíos epistemológicos y sociales de la enseñanza mediante cambio conceptual. Enseñanza de las Ciencias, 13(1), pp. 3-14.

FERNÁNDEZ, I. (1995). La transformación de las concepciones espontáneas sobre la ciencia. Tesis de tercer ciclo (no publicada). Universitat de València.

FURIÓ, C. (1994). La enseñanza-aprendizaje de las ciencias como investigación: un modelo emergente, en Proceedings International Conference «Science and Mathematics Education for the 21 st. century: Towards innovatory approaches», volumen I, p. 159-188. Concepción, Chile: Universidad de Concepción.

FURIÓ, C. y GIL, D. (1978).El programa-guía: una propuesta para la renovación de la didáctica de la física y química. València: ICE de la Universitat de València.

FURIÓ C., ITURBE J. y REYES, J.V. (1994). La résolution de problemes comme recherche. Aster, 19, pp. 87-102.

GABEL, D.L. (ed.) (1994). Handbook of Research on Science Teaching and Learning. Nueva York: MacMillan Pub. Co.

GARRETT, R., SATTERLY, D., GIL, D. y MARTÍNEZTORREGROSA, J. (1990). Turning exercises into problems: an experimental study with teachers in training. International Journal of Science Education, 12 (1), pp. 1-12

GENÉ, A. (1986). Transformación dels treballs pràctics de biología: una proposta teòricament fonamentada. Tesis doctoral. Barcelona: Facultat de Biologia de la Universitat de Barcelona.

GIL, D. (1983). Tres paradigmas básicos en la enseñanza de las ciencias. Enseñanza de las Ciencias, 1(1), pp. 26-33.

GIL, D. (1986). Emergencia de un nuevo paradigma de enseñanza-aprendizaje de las ciencias, en Del Río, Pablo (ed.). II Jornadas Inernacionales organizadas por Infancia y Aprendizaje.
GIL, D. (1991). ¿Qué han de saber y saber hacer los profesores de ciencias? Enseñanza de las Ciencias, 9(1), pp. 69-77.

GIL, D. (1993) Contribución de la historia y filosofía de las ciencias al desarrollo de un modelo de enseñanza-aprendizaje como investigación. Enseñanza de las Ciencias, 11(2), pp. 197-212.

GIL, D. (1993b). Psicología educativa y didáctica de las ciencias: los procesos de enseñanza-aprendizaje de las ciencias como lugar de encuentro. Infancia y aprendizaje, pp. 62-63.

GIL, D. (1994). Diez años de investigación en didáctica de las ciencias: realizaciones y perspectivas. Enseñanza de las Ciencias, 12(2), pp. 154-164.

GIL, D. (1997). Las concepciones docentes espontáneas sobre la ciencia como obstáculo para la renovación de la enseñanza de las ciencias, en Ospina H.F. y López, L. (comps.), Pedagogías constructivistas. Pedagogías activas y desarrollo humano. Bogotá: Cooperativa Editorial Magisterio.

GIL, D., CARRASCOSA, J. FURIÓ, C. y MARTÍNEZTORREGROSA, J. (1991). La enseñanza de las ciencias en la educación secundaria. Barcelona: Horsori.

GIL, D., DUMAS-CARRÉ, A., CAILLOT, M., MARTÍNEZ TORREGROSA, J. y RAMÍREZ, L. (1989). La resolución de problemas de lápiz y papel como actividad de investigación. Investigación en la escuela, 6, pp. 3-20.

GIL, D. y MARTÍNEZ-TORREGROSA, J. (1983). A model for problem-solving in accordance with scientific methodology. European Journal of Science Education, 5(4), pp. 447-455.

GIL, D. y MARTÍNEZ-TORREGROSA, J. (1987). Los programas-guía de actividades: una concreción del modelo constructivista de aprendizaje de las ciencias. Investigación en la escuela, 3, pp. 3-12.

GIL, D., MARTÍNEZ TORREGROSA, J. y SENENT, F. (1988). El fracaso en la resolución de problemas: una investigación orientada por nuevos supuestos. Enseñanza de las Ciencias, 6(2), pp. 131-146.

GIL, D. y PAYÁ, J. (1988). Los trabajos prácticos de física y química y la metodología científica. Revista de Enseñanza de la Física, 2(2), pp. 73-79.

GIL, D. y PESSOA, A. (1994). Formación del profesorado de las ciencias. Madrid Editorial Popular.

GIL, D. y VALDÉS, P. (1995). Contra la distinción clásica entre «teoría», «prácticas experimentales» y «resolución de problemas»: el estudio de las fuerzas elásticas como ejemplo ilustrativo. Didáctica de las ciencias experimentales y sociales, 9, pp. 3-25.

GIL, D. y VALDÉS, P. (1997). La resolución de problemas de física: de los ejercicios de aplicación al tratamiento de situaciones problemáticas. Revista de Enseñanza de la Físi$c a$. (Aceptado para publicación).

GUISASOLA, J. (1996). Análisis crítico de la enseñanza de la electrostática en el bachillerato y propuesta alternativa de orientación constructivista. Tesis doctoral. Universidad del País Vasco.

GOFFARD, M. (1990). Modes de travail pédagogiques et résolution de problèmes en Physique. Tesis doctoral. Universidad de París.

GONZÁLEZ, E. (1994). Las prácticas de laboratorio en la formación del profesorado de física. Tesis doctoral. Universitat de València. 
HODSON, D. (1985). Philosophy of science, science and science education. Studies in Science Education, 12, pp. 25-57.

HODSON, D. (1992). In search of a meaningful relationship: an exploration of some issues relating to integration in science and science education. International Journal of Science Education, 14(5), pp. 541-566.

HODSON, D. (1992b). Assessment of practical work: some considerations in Philosophy of Science. Science \& Education, 1, pp. 115-144.

HODSON, D. (1993). Philosophic stance of secondary school science teachers, curriculum experiences, and children's understanding of science: some preliminary findings. Interchange, 24(1-2), pp. 41-52.

KLOPFER, L.E. (1983). Research and the crisis in science education. Science Education, 67(3), pp. 283-284.

LAZAROWITZ, R. y TAMIR, P. (1994). Research on using laboratory instruction in science, en Gabel, D.L. (ed.). Handbook of Research on Science Teaching and Learning Nueva York: MacMillan Pub Co.

McDERMOTT, L.C. et al. (1996). Physics by inquiry. Nueva York: John Wiley \& Sons, Inc.

MALONEY, D.P. (1994). Research on problem solving: Physics, en Gabel, D.L. (ed.). Handbook of Research on Science Teaching and Learning. Nueva York: MacMillan Pub Co.

MARTÍNEZ-TORREGROSA, J. (1987). La resolución de problemas de física como investigación: un instrumento de cambio metodológico. Tesis doctoral. Facultad de Físicas. Universidad de Valencia.

MARTÍNEZ-TORREGROSA, J. et al. (1993). La búsqueda de la unidad (La estructura de todas las cosas). Libro del profesor y libro del alumno. Alicante: Aguaclara.

MARTÍNEZ-TORREGROSA, J. et al. (1995). El movimiento de todas las cosas. Alicante: Aguaclara.

MEICHSTRY, Y. (1993). The impact of science curricula on students views about the nature of science. Journal of Research in Science Teaching, 39(5), pp. 429-443.

MILLAR, R. y DRIVER, R. (1987). Beyond processes. Studies in Science Education, 14, pp. 33-62.

NATIONAL RESEARCH COUNCIL (1995). National Science Education Standards. Washington DC: National Academy Press.

OÑORBE, A. (1993). Análisis de dificultades en la enseñanzaaprendizaje de resoluciones de problemas de física y química. Tesis doctoral. Universidad de Alcalá de Henares.

OROZCO, A. (1995). El problema de las concepciones espontáneas sobre la ciencia. Tesis de tercer ciclo (no publicada). Universitat de València.

OSBORNE, R. y WITTROK, M. (1985). The generative learning model and its implications for science education. Studies in Science Education, 12, pp. 59-87.
PAYÁ, J. (1991). Los trabajos prácticos en física y química: un análisis crítico y una propuesta fundamentada. Tesis doctoral. Universitat de València.

PFUNDT, H. y DUIT, R. (1993). Bibliography: Students' alternative frameworks and science education. Kiel, Alemania: INP at the University of Kiel.

PORLÁN, R. (1993). Constructivismo y escuela. Hacia un modelo de enseñanza-aprendizaje basado en la investigación. Sevilla: Díada.

PORLÁN, R. y MARTÍN, R. (1996). Relaciones entre la historia y la filosofia de la ciencia y la enseñanza de las ciencias. Alambique, 8, pp. 23-32

POSNER, G.J. et al. (1982). Accommodation of a scientific conception: towards a theory of conceptual change. Science Education, 66, pp. 211-227.

PRAIA, J. y CACHAPUZ, F. (1994). Un análisis de las concepciones acerca de la naturaleza del conocimiento científico de los profesores portugueses de la enseñanza secundaria. Enseñanza de las Ciencias, 12(3), pp. 350-354.

RAMÍREZ, L. (1990). La resolución de problemas de física y química como investigación en la enseñanza media, un instrumento de cambio metodológico. Facultad de Ciencias Químicas. Universidad Autónoma de Barcelona.

REYES, J.V. (1991). La resolución de problemas de química como investigación: una propuesta didáctica basada en el cambio metodológico. Tesis doctoral. Universidad del País Vasco.

SALINAS, J. (1994). Las prácticas de física básica en laboratorios universitarios. Tesis doctoral. Universitat de València.

SALINAS, J. y COLOMBO DE CUDMANI, L. (1992). Los laboratorios de física de ciclos básicos universitarios instrumentados como procesos colectivos de investigación dirigida. Revista de Enseñanza de la Física, 5(2), pp. 10-17.

SANMARTÍ, N., MAURI, T., IZQUIERDO, M. y GÓMEZ, I. (1990). Los procedimientos. Cuadernos de Pedagogía, 180, pp. 28-32.

SOLBES, J. (1986). La introducción de los conceptos básicos de física moderna. Tesis doctoral. Facultat de Ciències Físiques de la Universitat de València.

SOLOMON, J. (1991). Teaching about the nature of science in the British National Curriculurn. Science Education, 75 (1), pp. 95-103.

TIBERGHIEN, A. (1983). La investigación en un laboratorio de didáctica de las ciencias físicas. Enseñanza de las Ciencias, 1(3), pp. 187-192.

VALDÉS, R. y VALDÉS, P. (1994). Utilización de los ordenadores en la enseñanza de la física. Revista Española de Física, 8(4), pp. 50-52.

VIENNOT, L. (1989). L'enseignement des sciences physiques object de recherche. Bulletin de l'Union des Physiciens, 716, pp. 899-910.

WHEATLEY, G.H. (1991). Constructivist perspectives on Science and Mathematics learning. Science Education, 75(1), pp. 9-21. 